Uso de Indicadores de Patentes como Metodologia para Avaliação do Desenvolvimento da Tecnologia de Extração Supercrítica

\author{
Machado, B. A. S. *; Costa, S. S.; Silva, R. P. D.; Alves, A. R. C.; Guarieiro, \\ L. L. N.; Padilha, F. F.
}

Rev. Virtual Quim., 2016, 8 (4), 1079-1093. Data de publicação na Web: 29 de julho de 2016

http://rvq.sbq.org.br

\title{
Use of Patent Indicators as a Methodology for Evaluating the Development of Supercritical Extraction Technology
}

Abstract: The use of science and technology indicators that plan investments in research, development and innovation has been growing in recent years. This article aimed to evaluate the scientific and technological indicators, through research related to patent applications and spending on RD\&l carried out by the sponsor countries of supercritical extraction technology. Survey was carried out in the online databases for European office Espacenet (EP), by collecting, processing and analysis of information extracted from patent documents. The researched technology is mainly concentrated in developed countries, which have the largest investments in RD\&I in recent years.

Keywords: Indicators; patents; supercritical extraction; innovation.

\section{Resumo}

A utilização de indicadores científicos e tecnológicos que traçam os investimentos em pesquisa, desenvolvimento e inovação vem crescendo nos últimos anos. Este artigo teve como objetivo avaliar os indicadores tecnológicos e científicos, por meio da pesquisa relacionada ao depósito de patentes e gastos com PD\&I realizados pelos países detentores da tecnologia de extração supercrítica. A pesquisa foi realizada nas bases de dados online do escritório europeu Espacenet (EP), com a coleta, tratamento e análise das informações extraídas dos documentos de patentes. A tecnologia pesquisada se encontra principalmente concentrada em países desenvolvidos, os quais possuem os maiores investimentos financeiros em PD\&I nos últimos anos.

Palavras-chave: Indicadores; patentes; extração supercrítica; inovação.

* SENAI CIMATEC, Faculdade de Tecnologia, Centro Integrado de Manufatura e Tecnologia, Área Tecnológica de Alimentos e Bebidas, CEP 41650-010, Salvador-BA, Brasil.

M brunam@fieb.org.br

DOI: $\underline{10.21577 / 1984-6835.20160077}$ 


\title{
Uso de Indicadores de Patentes como Metodologia para Avaliação do Desenvolvimento da Tecnologia de Extração Supercrítica
}

\author{
Bruna Aparecida S. Machado, ${ }^{a, b, *}$ Samantha S. Costa, ${ }^{a}$ Rejane P. D. \\ Silva, ${ }^{a, c}$ Aline R. C. Alves, ${ }^{a}$ Lilian L. N. Guarieiro, ${ }^{\text {d,e,f }}$ Francine F. Padilha $^{b}$ \\ ${ }^{a}$ SENAI CIMATEC, Faculdade de Tecnologia, Centro Integrado de Manufatura e Tecnologia, \\ Área Tecnológica de Alimentos e Bebidas, CEP 41650-010, Salvador-BA, Brasil. \\ ${ }^{\mathrm{b}}$ Universidade Tiradentes, Biotecnologia, CEP 49032-490, Aracaju-SE, Brasil. \\ ' Universidade Federal da Bahia, Faculdade de Farmácia, Programa de Pós Graduação em \\ Ciência de Alimentos, CEP 40170-290, Salvador-BA, Brasil. \\ ${ }^{d}$ SENAI CIMATEC, Faculdade de Tecnologia, Centro Integrado de Manufatura e Tecnologia, \\ Área Mobilidade, CEP 41650-010, Salvador-BA, Brasil. \\ e Universidade Federal da Bahia, Instituto Nacional de Ciência e Tecnologia em Energia e \\ Ambiente, CEP 40170-290, Salvador-BA, Brasil. \\ ${ }^{\text {f }}$ SENAI CIMATEC, Faculdade de Tecnologia, Centro Integrado de Manufatura e Tecnologia, \\ PRH\%\% ANP/MCTI, CEP 41650-010, Salvador-BA, Brasil. \\ * brunam@fieb.org.br
}

\section{Introdução \\ 2. Aspectos Metodológicos \\ 3. Resultados e Discussão \\ 4. Conclusão}

\section{Introdução}

Desde o final da Segunda Guerra Mundial, a crescente demanda por recursos e espaço para atividades de Ciência e Tecnologia (C\&T), tanto nos países como nas empresas, despertou a atenção de analistas e estudiosos. $O$ interesse por indicadores quantitativos vem crescendo nos últimos anos com o objetivo de auxiliar os especialistas e autoridades governamentais para o planejamento de políticas e tomadas de decisões no setor tecnológico. ${ }^{1}$ Destaca-se que a importância atual na utilização de indicadores científicos e tecnológicos que traçam os investimentos em Pesquisa, Desenvolvimento e Inovação (PD\&I) é devido principalmente à recente necessidade de se obter um direcionamento e monitoramento dos esforços tecnológicos e científicos. A correlação desses indicadores com os 
investimentos em PD\&l é uma ferramenta eficiente para avaliar os esforços inovativos dos países, empresas e universidades em diferentes campos tecnológicos.

Um dos indicadores para o progresso da C\&T e suas perspectivas futuras é verificar quanto os países investem em cada área de interesse. Os investimentos avaliados são os recursos financeiros destinados ao pagamento de recursos humanos qualificados, construção e manutenção de infraestrutura, equipamentos de laboratórios e gastos operativos com insumos para a pesquisa, viagens técnicas, publicações e comunicação. ${ }^{2}$ Neste contexto, destaca-se como ferramenta de medição os métodos presentes no Manual Frascati, que tem por objeto definir e estruturar os dispêndios e recursos humanos alocados em PD\&I. Além de definir detalhadamente essas atividades, orienta na distinção de outras que podem ser confundidas com PD\&l, apresenta as principais classificações setoriais e funcionais dos dados de PD\&l e discorre sobre a medição e classificação dos recursos humanos e dispêndios nesta atividade. $O$ manual detalha ainda os métodos de medição, principalmente no que se refere às estimativas e aos ajustes necessários. Por fim, dedica um capítulo à comparação desses dados com outras variáveis econômicas e comparação internacional do dispêndio em PD\&I. ${ }^{3}$

Dados como publicações bibliométricas e patentes são comumente utilizados para avaliar o cenário científico e tecnológico mundial, assim como classificar empresas que fazem uso de PD\&I. De acordo com o Manual de Frascatti, ${ }^{4}$ as patentes representam em maior medida o produto da investigação tecnológica e empresarial, uma vez que protegem os conhecimentos com potencial de interesse econômico. Com relação à publicação de artigos científicos, representa maior aproximação entre o produto e a pesquisa acadêmica. ${ }^{5,6}$ Diante de tal definição, o foco desse estudo está baseado na utilização dos documentos de patentes como indicadores para a avaliação tecnológica do processo de Extração
Supercrítica.

Extração com fluido supercrítico (EFS) é uma operação que explora o poder de solvatação dos fluidos à temperatura e pressão acima de seus valores críticos para extrair ou separar componentes de uma amostra. A aplicação de EFS está baseada na observação experimental de que muitos gases aumentam seu poder de dissolução, quando comprimidos acima do ponto crítico. ${ }^{7-10}$ A importância do uso desses fluidos foi descoberta e começou a ser utilizada no final do século XIX, Hannay e Hogard, em 1879, ${ }^{11}$ propuseram o modelo de que o aumento simultâneo na pressão e na temperatura de certas substâncias químicas aumentava sua solubilidade, facilitando o processo de extração. Em 1906, Buncher ${ }^{11}$ confirmou esse modelo através de experimentos realizados, sistematizando melhor o processo. Bundschuh, ${ }^{11}$ em 1986, verificou que os fluidos nestas condições têm suas propriedades como densidade, difusividade e viscosidade, com características de gases e líquidos simultaneamente. Torna-se importante destacar que embora os fluidos supercríticos tenham sido conhecidos há mais de um século, estes ainda vêm sendo tratados como uma descoberta recente. ${ }^{12} \mathrm{~A}$ utilização desta técnica de extração como instrumento analítico e preparativo somente ganhou destaque nos últimos 30 anos, o que é confirmado pelo elevado número de artigos publicados e patentes depositadas nesta área em todo o mundo.

A elevada utilização de solventes orgânicos nos diferentes processos industriais, tais como, extração de óleos e gorduras, obtenção de compostos funcionais bioativos, remoção de metais pesados, processamento de polímeros, produção de combustíveis, entre outras, representa um problema discutido globalmente, devido aos danos causados ao meio ambiente. Em virtude desse cenário, foi introduzido em 1987 o Protocolo de Montreal, e em 1997 o Protocolo de Kyoto, que tiveram como objetivo principal restringir ou eliminar a produção e utilização de solventes que 
causem danos à camada de ozônio. ${ }^{13,14} \mathrm{O}$ grande interesse da comunidade científica e do setor industrial pela EFS está diretamente relacionado às restrições ao uso de solventes orgânicos, tanto nos processos preparativos de amostras utilizados nas diversas indústrias, junto à maior consciência ecológica no uso destes em diferentes métodos de análise envolvendo extração.

A extração por tecnologia supercrítica possui vantagens e limitações quando comparada às técnicas clássicas, tais como a extração líquido-líquido, maceração e extração com solvente orgânico. ${ }^{15-17}$ Uma das principais vantagens da utilização da tecnologia de extração com fluidos supercríticos é decorrente principalmente do uso do dióxido de carbono como solvente extrator, substância esta que possui propriedades bastante superiores à maioria dos solventes orgânicos.

Há atualmente uma grande variedade de compostos que podem ser usados como solventes supercríticos, como por exemplo, a água, o benzeno, tolueno, etano, etanol, dióxido de carbono $\left(\mathrm{CO}_{2}\right)$, entre outros. Dentre os compostos utilizados em EFS o $\mathrm{CO}_{2}$ é o solvente mais utilizado, devido principalmente às suas características de baixo custo, ser facilmente disponível em alta pureza, não ser tóxico, inflamável e explosivo. Outra vantagem apresentada pelo $\mathrm{CO}_{2}$ é sua facilidade de ser removido após o processo de extração por ser um gás à temperatura e pressão ambientes. ${ }^{18}$ Apesar das vantagens apresentadas pela utilização da tecnologia de EFS, deve-se destacar que a principal limitação do uso desta tecnologia está relacionada ao elevado custo dos equipamentos disponíveis comercialmente quando comparados a sistemas convencionais de extração, principalmente quando se trata de plantas industriais.

Grande parte da importância dada atualmente para a utilização de fluidos supercríticos em processos de extração está extremamente vinculada à necessidade em substituir solventes orgânicos, responsáveis por grandes problemas ambientais, e também devido à tendência de um rápido aumento dos custos industriais devido à elevação de gastos energéticos advindo da crise do petróleo. Diante do cenário de grande importância na utilização desta técnica como método extrativo, preparativo e analítico para diversas substâncias, e ainda, o processo inovativo associado a esta tecnologia, este artigo teve como objetivo avaliar os indicadores tecnológicos e científicos, por meio da pesquisa relacionada ao depósito de patentes e investimentos financeiros em PD\&l realizados pelos países detentores da tecnologia de extração supercrítica.

\section{Aspectos Metodológicos}

Para a pesquisa da tecnologia de interesse, protegida ou descrita em documentos de patentes (Figura 1), foi desenvolvida uma estratégia de busca que combinou a utilização de códigos da Classificação Internacional de Patentes (CIP), nas quais os documentos relativos a esta tecnologia de extração supercrítica foram limitados as classificações: B01D 11/00; B01D 11/02; B01D 11/04; B01D 15/40; associados a um conjunto de palavras-chave (extração supercrítica, fluidos supercríticos, extração com fluidos supercríticos, dióxido de carbono supercrítico, temperatura crítica, pressão crítica) que representam as formas com as quais esta tecnologia poderia ser identificada nos documentos. ${ }^{19-20}$ A pesquisa foi realizada na base de patentes do escritório europeu (Espacenet - EP), que compila um acervo de patentes depositadas e publicadas em mais de 90 países. 


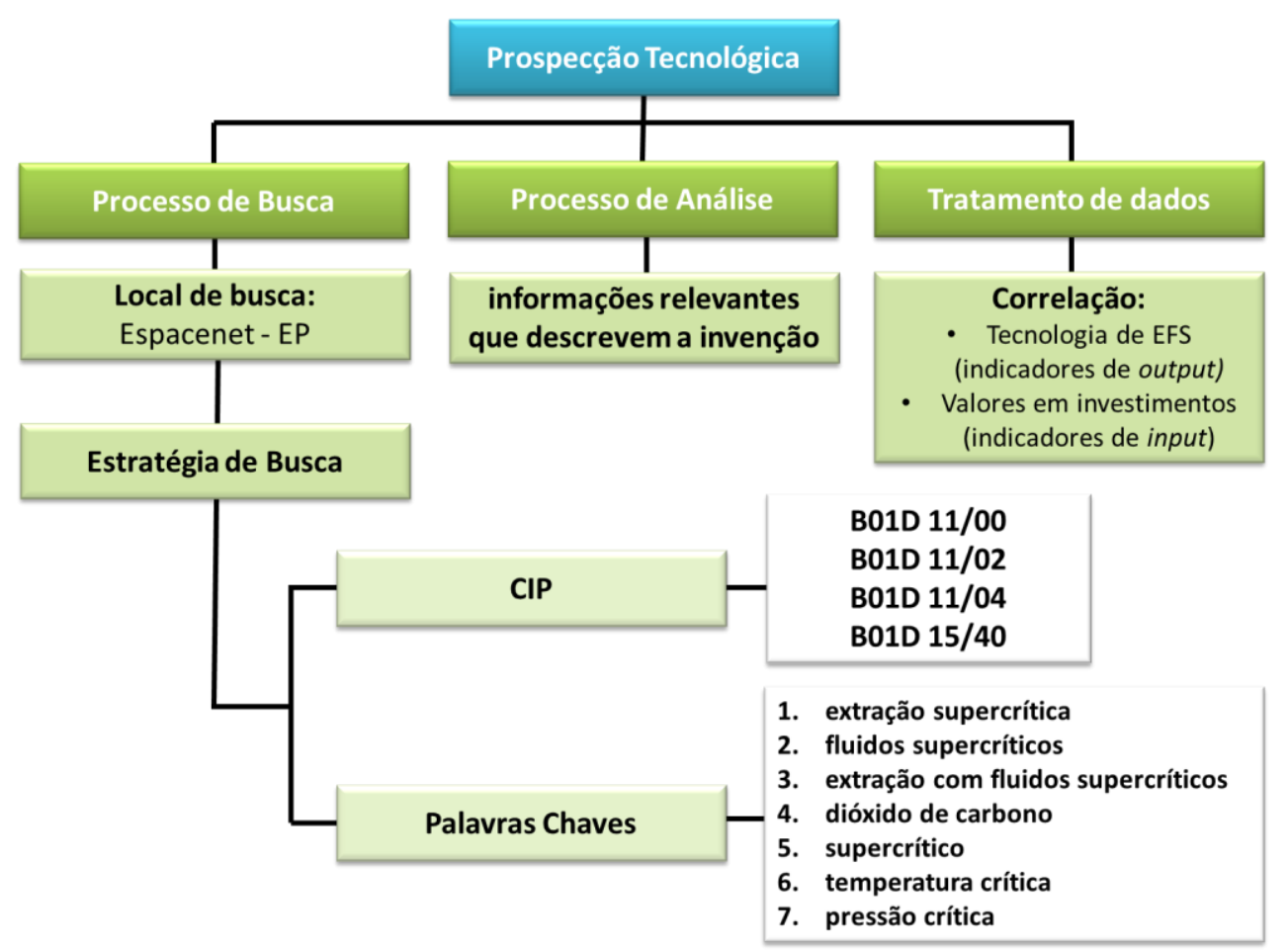

Figura 1. Fluxograma de Prospecção Tecnológica

O estudo foi realizado por meio de coleta, tratamento e análise das informações extraídas dos documentos de patentes (Figura 1). O termo documento de patente abrange pedidos de patente publicados ou patentes concedidas. Para interpretar as informações da tecnologia patenteada, cada documento foi analisado e deles extraídas as informações relevantes que descrevem a invenção e expressos em gráficos. Os resultados encontrados em relação aos documentos de patentes (indicadores de output) relacionados à tecnologia de extração supercrítica foram correlacionados com valores em investimentos em PD\&I de alguns países, sendo estes considerados como indicadores de input. Essa correlação foi feita com base nas orientações contidas no Manual de Frascati (1983/2002) em relação aos conceitos e aplicações dos recursos relacionados à $\mathrm{PD \& l}{ }^{4}{ }^{4} \mathrm{Em}$ relação às patentes, o Manual de Oslo ${ }^{4}$ as definem e classificam como indicadores de inovação. Entretanto, neste estudo, com base em informações extraídas de outros trabalhos e opiniões de diferentes autores, consideraram-se as patentes como um indicador de esforço inovativo por países, empresas e indivíduos.

\section{Resultados e Discussão}

A partir da pesquisa realizada no banco de dados do escritório europeu, (Espacenet) foram identificados 3.819 registros de documentos de patentes que faziam relação com as palavras-chaves e códigos pesquisados. Apesar do grande universo de dados identificados, ressalta-se que este número não representa o total de invenções protegidas nesta área, já que um mesmo documento de patente pode ser depositado em diferentes países, com o objetivo de garantir o direito de exclusividade aos seus inventores nos mercados considerados mais relevantes, uma vez que o direito da patente é territorial. Além disso, documentos que apenas faziam referência à tecnologia de interesse também foram coletados com a estratégia de busca utilizada, sendo, entretanto, posteriormente excluídos, tendo em vista a não relevância dos mesmos para a 
discussão dos resultados. O universo de documentos de patentes que se relacionavam com a tecnologia de EFS foi composto por 815 documentos depositados entre os anos de 1974 e 2014 (Figura 2).

A primeira patente depositada sobre EFS identificada neste estudo foi no ano de 1974 (GB1481958), de titularidade da Indústria Coal, tendo o Reino Unido como país de origem. A invenção revela o processo que envolve a extração supercrítica e hidrogenação catalítica de compostos derivados do carvão. Steffani ${ }^{21}$ reporta que a primeira proposta de aplicação prática da EFS ocorreu em 1943, para o desasfaltamento de petróleo. Industrialmente, a EFS teve seu início na Alemanha, em 1978, com o processo da remoção da cafeína do café. ${ }^{22-24}$

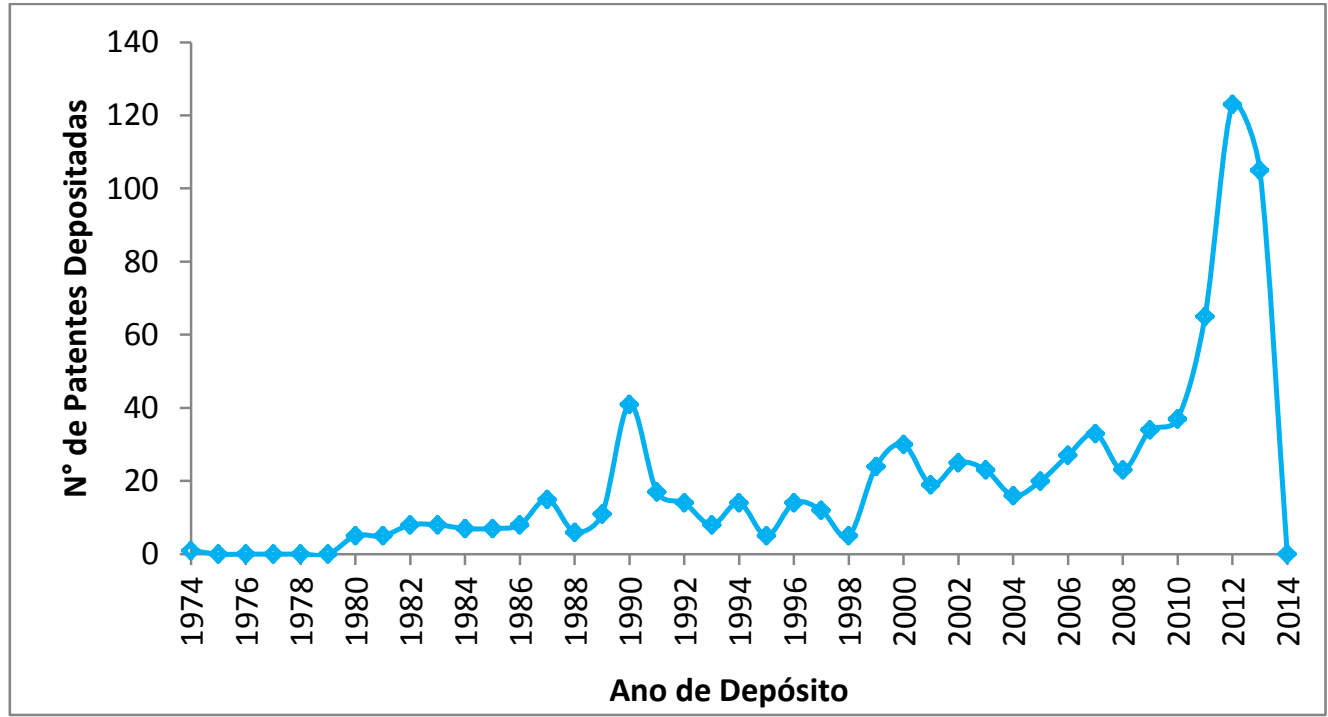

Figura 2. Evolução anual de depósitos de patentes sobre a tecnologia de extração supercrítica entre 1974 e 2014

A partir dos dados apresentados na Figura 2, identificou-se que, entre os anos de 1974 e 1988, (com 70 documentos de patentes depositados) as patentes relacionam-se principalmente com a tecnologia básica da tecnologia de extração supercrítica, ou seja, aplicação de fluidos em condições não otimizadas e de maneira simplificada para obtenção de compostos de interesse de amostras alimentícias e produtos naturais farmacêuticos. Após esse período, começaram a surgir as patentes relacionadas às aplicações da tecnologia de extração supercrítica em outras áreas (petróleo, veterinária, polímeros, química), bem como as técnicas mais modernas do processo extrativo, com parâmetros otimizados, destacando-se ainda a utilização desta técnica para a determinação de pesticidas e componentes traços na área de alimentos e o aperfeiçoamento dos processos técnicos na indústria farmacêutica e cosmética. Entre os anos de 2000 e 2013, é encontrado o maior número de documentos de patentes depositados sobre EFS, com 580 depósitos, e isso pode estar relacionado com as políticas mundiais que incentivam cada vez mais a diminuição da utilização de solventes orgânicos nas diferentes áreas industriais. Nesse período, é observado um constante crescimento no número de depósitos de patentes, com destaque para os anos de 2012 e 2013, nos quais foram identificados 123 e 105 depósitos, respectivamente. Destaca-se ainda que o total de invenções identificados em 2013 não representa o valor real, tendo em vista o período de sigilo proposto na legislação patentária, onde os documentos de patentes só são publicados após 18 meses da data do depósito. E, ainda 
por essa razão, não foi identificado nenhum documento depositado em 2014.

Outra análise realizada no estudo foi relativa aos titulares das patentes depositadas sobre a tecnologia de EFS (Figura 3). Tal análise é importante para avaliar como se encontra distribuído os investimentos em PD\&I responsáveis por gerar novos produtos ou novos processos em relação à tecnologia pesquisada, ou seja, se é o setor industrial, governamental ou universidades que mais investem seus recursos para o desenvolvimento de inovações. Do total de documentos depositados, 49,4\% (403 documentos depositados) são de titularidade de empresas, revelando assim que, para a tecnologia pesquisada, os maiores investimentos são realizados pelo setor industrial. Os pedidos de patentes solicitados por inventores independentes representam 26,9\% (219 documentos depositados) e para as universidades e centros de pesquisa identificou-se um percentual de 23,7\% (193 documentos depositados).

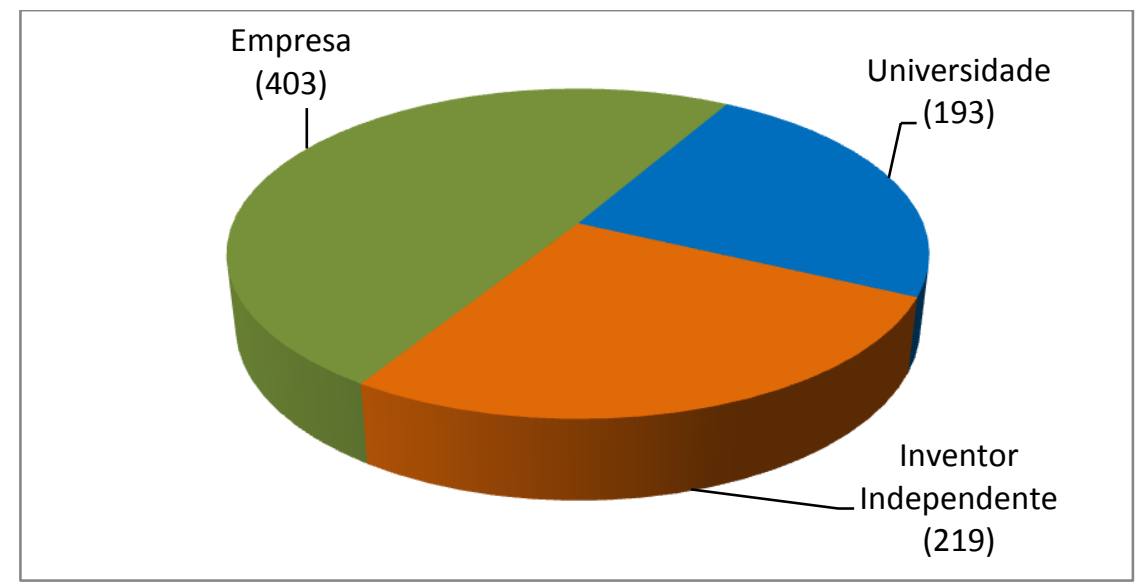

Figura 3. Número de patentes identificadas, por tipo de depositante, relacionados à tecnologia de extração por fluidos supercríticos

A análise dos documentos de patentes depositados, no que diz respeito aos países nos quais se originou a tecnologia patenteada (identificação do país de origem do depositante), revela que esta tecnologia se encontra principalmente centralizada em países desenvolvidos, com destaque para países asiáticos. A Figura 4 apresenta o número de documentos de patentes depositados no escritório europeu, por país de origem/região, que não estão em sigilo até o momento da pesquisa (1974 a 2014).

A China, Estados Unidos, Coreia do Sul e Japão são responsáveis pelo domínio de $77,8 \%$ da tecnologia patenteada sobre EFS
(634 documentos depositados), revelando assim o grande interesse desses países pelo desenvolvimento de novos processos e produtos relacionados à EFS. Apesar da China ser atualmente o país com o maior número de patentes depositadas na área, a maioria das plantas industriais em funcionamento que realizam $o$ processo de extração supercrítica em amostras de tabaco, café, lúpulo, aromas, fármacos de origens botânicas, entre outros, estão localizadas nos Estados Unidos. Ressalta-se que os Estados Unidos e Alemanha foram os pioneiros na implantação de plantas industriais de extração com fluido supercrítico. 


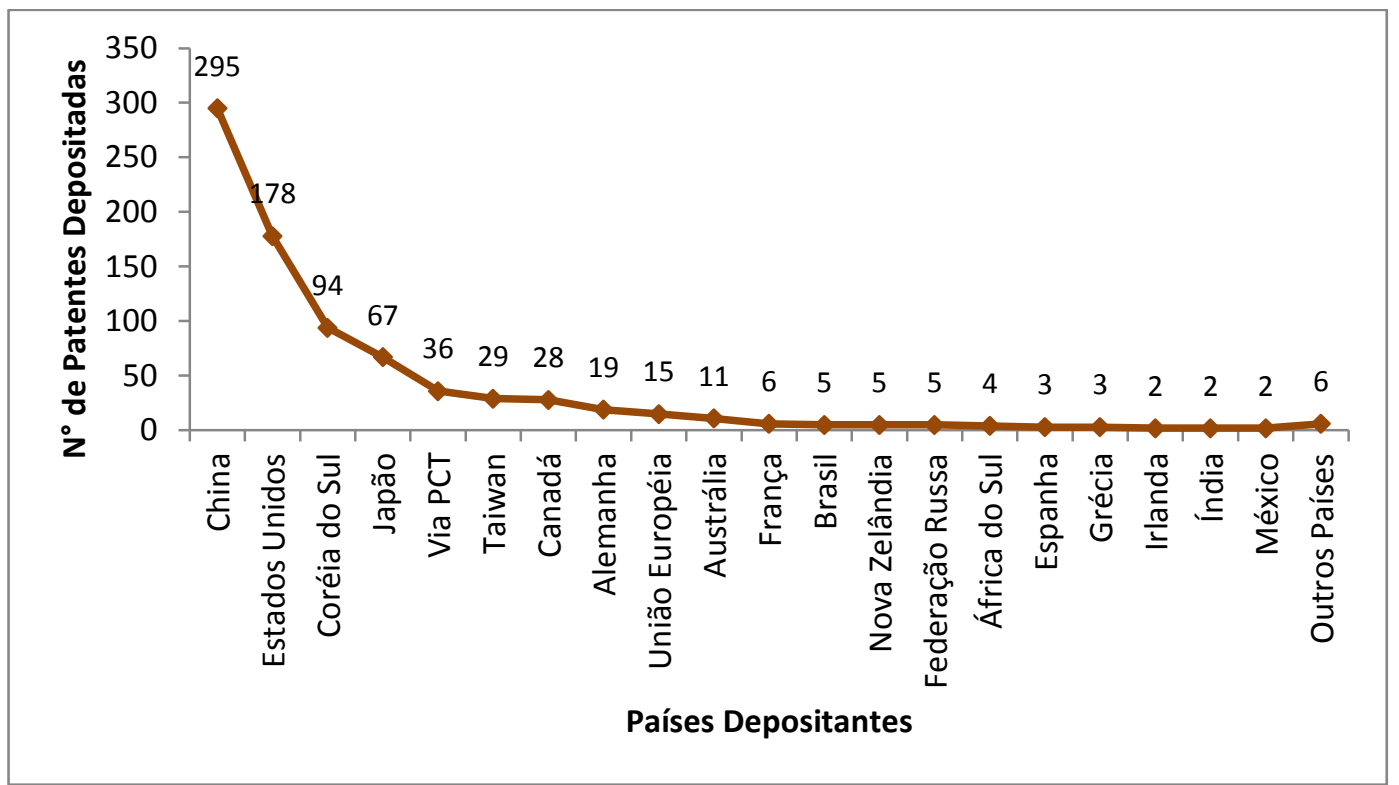

Figura 4. Depósitos de patentes relacionadas à tecnologia de extração com fluidos supercríticos por país de origem/região dos depositantes entre 1974 e 2014 (PCT: Patent Cooperation Treaty)

Correlacionando a Figura 2 com a Figura 4, foi possível identificar que, até 0 ano de 1990, o principal detentor da tecnologia de extração supercrítica era os Estados Unidos, que além de ocupar a primeira posição no número de depósitos de patentes, possuía $41 \%$ das patentes depositadas no período. No mesmo período, a China ocupava a quarta posição. Entretanto, a partir da década de 90 , observou-se um elevado crescimento de patentes chinesas, principalmente depositadas por universidades. A mudança nesse cenário pode estar relacionada a dois motivos. Maiores investimentos na década de 80 em educação no país, incluindo nas políticas públicas os investimentos em PD\&I para o fortalecimento e desenvolvimento econômico e social, e ainda, o estímulo à cultura da propriedade intelectual, através principalmente da proteção com depósito de patentes nas universidades. A grande maioria dos documentos de patentes chineses se trata da obtenção de compostos bioativos de matrizes naturais de origem botânica, em virtude principalmente do grande conhecimento e aplicação da medicina tradicional.
Avaliando o caso brasileiro, foram identificados nesse estudo apenas cinco documentos de patentes depositados na área de EFS (Figura 4). Apesar de ser uma tecnologia de grande interesse em países desenvolvidos e em crescente desenvolvimento, no que diz respeito ao depósito de patentes, o Brasil posiciona-se como um país pouco representativo. Isso não é justificado apenas pelo pequeno número de depósitos pelo Brasil, mas também pelo pouco interesse dos depósitos de não residentes. Na base de dados do Instituto Nacional de Propriedade Industrial, que compila 0 acervo de todas patentes depositadas no Brasil, foram identificadas apenas 10 patentes relacionadas à EFS, sendo 05 documentos depositados por não residentes (03 de titularidade dos Estados Unidos, 01 de titularidade da Índia e 01 de titularidade da França). Neste cenário de patentes, o Brasil mostra-se como um mercado pouco promissor em relação à área tecnológica pesquisada, não atraindo, portanto, 0 interesse de empresas, universidades e centro de pesquisas internacionais em proteger suas invenções no mercado brasileiro. Torna-se importante 
ressaltar que inúmeros pesquisadores de universidades e centros de pesquisa nacionais têm se destacado desenvolvendo processos de extração, fracionamento e purificação envolvendo fluidos supercríticos, além de reações químicas em meio supercrítico, cristalização com solvente supercrítico e processamento de polímeros, com publicações internacionais importantes na área. Entretanto, o resultado obtido nas pesquisas não vem sendo aplicado através da proteção por patentes. O documento de patente brasileiro envolvendo extração supercrítica mais recente é do ano de 2007.

$\mathrm{Na}$ Tabela 1 são apresentados os valores em investimentos em PD\&I dos principais países detentores da tecnologia de EFS, comparando com os investimentos realizados pelo Brasil. Nesta etapa do trabalho, tem-se a correlação entre indicadores de input, ou de investimento (gastos em PD\&I), com os indicadores de output, denominados também de produtos gerados com os investimentos (documentos de patentes). As informações foram coletadas no Relatório da OECD (Organization for Economic Co-Operation and Development) ${ }^{25}$ para a China, Estados Unidos, Japão e Coreia do Sul, e no MCTI (Ministério da Ciência, Tecnologia e Inovação) ${ }^{26}$ para o Brasil.

Com o objetivo de correlacionar os documentos de patentes depositados nesse setor tecnológico com o total geral de documentos patentes depositados pela China, Estados Unidos, Coreia do Sul e Brasil, na Tabela 2 é apresentada o número de patentes depositadas no escritório de marcas e patentes norte americano USPTO (United States Patent and Trademark Office) em 2010. Foi escolhido o escritório norte americano como fonte de informações de depósitos de patentes, já que este é o principal mercado de interesse internacional para a proteção das diferentes tecnologias pela maioria dos países.

Tabela 1. Valores em investimentos relacionados a PD\&I dos principais países detentores da tecnologia de extração com fluidos supercríticos (China, Estados Unidos, Coreia do Sul e Japão) e do Brasil em 2009

\begin{tabular}{cccccc}
\hline \multirow{2}{*}{$\begin{array}{c}\text { Tipos de } \\
\text { Indicadores }\end{array}$} & China $^{\mathbf{A}}$ & $\begin{array}{c}\text { Estados } \\
\text { Unidos }\end{array}$ & $\begin{array}{c}\text { Coreíses } \\
\text { Sul }^{\mathbf{A}}\end{array}$ & Japão $^{\mathbf{A}}$ & Brasil $^{\mathbf{B}}$ \\
\cline { 2 - 6 } & & & & & \\
\hline $\begin{array}{c}\text { Investimento } \\
\text { financeiro em PD\& } \\
\text { (milhões de dólares) }\end{array}$ & $154.147,36$ & $401.576,00$ & $53.184,86$ & $137.314,21$ & $23.708,00$ \\
$\begin{array}{c}\text { Aplicação do PIB em } \\
\text { PD\&I (\%) }\end{array}$ & 1,70 & 2,90 & 3,74 & 3,36 & 1,25 \\
$\begin{array}{c}\text { Aplicação do PIB em } \\
\text { pesquisa básica (\%) } \\
\text { Recursos Humanos } \\
\text { inseridos em PD\&l }\end{array}$ & 0,08 & 0,55 & 0,64 & 0,42 & 0,41 \\
\hline
\end{tabular}

${ }^{\mathrm{A}} \mathrm{OECD}{ }^{25} ;{ }^{\mathrm{B}} \mathrm{MCT}{ }^{26}$ 
Tabela 2. Número de documentos de patentes depositados no escritório de marcas e patentes norte americano USPTO (United States Patent and Trademark Office) em 2010 pela China, Estados Unidos, Coreia do Sul, Japão e Brasil

\begin{tabular}{lc}
\hline \multicolumn{1}{c}{ País } & Número de documentos de patentes - USPTO \\
\hline China & 8.162 \\
Estados Unidos & 241.977 \\
Coréia do Sul & 26.040 \\
Japão & 84.017 \\
Brasil & 568 \\
\hline
\end{tabular}

$\operatorname{MCTI}(2012)^{26}$

Uma comparação de gastos em PD\&l, como porcentagem do Produto Interno Bruto (PIB) entre países, pode ser vista na Tabela 1 , na qual se constata que o Brasil gasta menos que as nações mais desenvolvidas. O Brasil gastou, em 2009, 1,25\% do PIB em PD\&I, enquanto que Coreia do Sul, Japão, Estados Unidos e China, gastaram 3,74\%, 3,36\%, $2,90 \%$ e $1,70 \%$, respectivamente. Observa-se que, para o caso brasileiro, o investimento em PD\&l é pequeno por parte do governo, quando comparado ao padrão de gastos ao de nações mais desenvolvidas. Em relação ao depósito de patentes relacionadas à tecnologia de EFS (Figura 4), apesar de a China possuir um percentual de gasto em relação ao PIB menor do que os Estados Unidos, Japão e Coreia do Sul, possui o maior número de patentes depositadas neste setor tecnológico. Entretanto, em relação ao número global de documentos de patentes depositados no USPTO em diferentes áreas tecnológicas, os investimentos em PD\&I realizados pelos países refletem no número de depósitos. Os maiores investimentos em PD\&l são realizados pelos Estados Unidos (401.576,00 milhões de dólares) e Japão (137.314,21 milhões de dólares), que possuem os maiores números de depósitos de patentes nas diferentes áreas tecnológicas. O Brasil, por sua vez, possui apenas 568 documentos de patentes depositados no USPTO, apresentando também o menor investimento em PD\&l (23.708,00 milhões de dólares), quando comparado aos países analisados neste estudo.

É importante destacar que não há como prever de maneira precisa se os gastos com PD\&l serão satisfatórios no futuro, devido principalmente à existência de fatores externos que influenciam direta e indiretamente no sucesso destes investimentos, tais como, as condições globais do mercado, investimentos em PD\&I por empresas, aplicação adequada destes investimentos em pesquisa, entre outros. De acordo com Queiroz, ${ }^{27}$ os gastos com PD\&l não podem ser avaliados da mesma forma que outros processos produtivos, pois o seu retorno para o país, empresa ou sociedade é considerado de médio e longo prazo. Dessa forma, analisando os investimentos em PD\&I, estes poderão gerar muitas vezes resultados negativos em curto prazo, pois antes que o produto final seja obtido, existem apenas gastos sem a geração de resultados/receitas, que neste estudo são considerados os documentos de patentes gerados a partir destes investimentos como forma de avaliar o esforço inovativo do país, ou seja, o seu desenvolvimento tecnológico.

A partir dos resultados apresentados nas Tabelas 1 e 2 e de acordo com Kim, ${ }^{28}$ em países industrializados, mais de $50 \%$ do crescimento econômico de longo prazo 
deriva de mudanças tecnológicas que promovem melhorias na produtividade e o lançamento de novos produtos, processos ou setores industriais inteiros. Como resultado desta política, os Estados Unidos vivenciou uma expansão nos investimentos em PD\&I até a década de 80. A partir desta década, as pressões para reduzir os déficits orçamentários e a destinação de recursos para outras finalidades fez com que os recursos de PD\&I ficassem escassos. O que ocorre também neste país é que o governo têm estimulado as universidades e os centros públicos de pesquisas a cooperar com o setor produtivo para reduzir a carga orçamentária com PD\&I. Sendo assim, novos modelos de negócios, a maioria deles originários do Japão, têm enfatizado a colaboração na cadeia de suprimentos visando compartilhar os custos e ganhos com PD\&I. ${ }^{29}$

É possível observar que os países cuja economia é considerada representativa em nível mundial, mas ainda se encontra em um processo de desenvolvimento e amadurecimento do sistema tecnológico, como é o caso da China e do Brasil, tem como características um mercado amplo, com consumidores de baixo poder aquisitivo, com taxas de PD\&I e atividades inovativas baixas, se comparadas aos dos países tecnologicamente desenvolvidos. ${ }^{30}$ Independente disso, quando é feita uma análise comparativa entre o número de patentes depositadas pelo Brasil e China relacionados à tecnologia de EFS, observa-se a discrepância de números (Figura 4, Tabela 1). Assim, no caso da tecnologia relacionada à extração supercrítica, a China apesar de possuir menores investimentos financeiros em PD\&I que os Estados Unidos, apresenta o maior número de patentes na área pesquisada.

Outra análise bastante importante realizada no estudo refere-se às áreas de aplicações das patentes desenvolvidas e depositadas (Figura 5). Atualmente, a extração com fluidos encontra aplicações industriais já implantadas ou em fase de estudo ou experimentação, nas mais diversas áreas. Nos últimos anos, a extração com fluido supercrítico conquistou posições expressivas em diversos setores das indústrias químicas, petroquímicas, farmacêuticas, de alimentos, de polímeros, entre outras. Em relação às aplicações dessa tecnologia referente aos indicadores de patentes, identificou-se que $35,8 \%$ dos documentos depositados estão inseridos na área de alimentos e agricultura. Resultados expressivos também foram encontrados para a aplicação dessa tecnologia nas áreas da indústria química e petroquímica, com $23 \%$ do total de documentos depositados, e na indústria farmacêutica, representada por 17\% dos documentos (Figura 5).

Novas aplicações da tecnologia de EFS vêm sendo desenvolvidas nos últimos anos, com pesquisas nas áreas de toxicologia, ambiental, têxteis, polímeros, entre outras. ${ }^{31-}$ 35 Ressalta-se que fluidos supercríticos apresentam viscosidade baixa como a de um gás, alta densidade como os líquidos $\mathrm{e}$ difusão intermediária entre gases e líquidos, variando com a sua densidade. ${ }^{36,37}$ São facilmente adaptáveis aos diversos processos de separação, que envolvem amplas faixas de temperatura e pressão. Tal característica permite que os fluidos supercríticos sejam utilizados para separar materiais termicamente instáveis (óleos e gorduras poliinsaturados, vitaminas, carotenoides, compostos antioxidantes), a baixas temperaturas, e ainda efetuar separações com pequenas variações de pressão, devido à alta compressibilidade e solubilidade exponencial. ${ }^{38,39}$ Estas características tornam estes fluidos bons substitutos para os solventes orgânicos em processos de extração. 


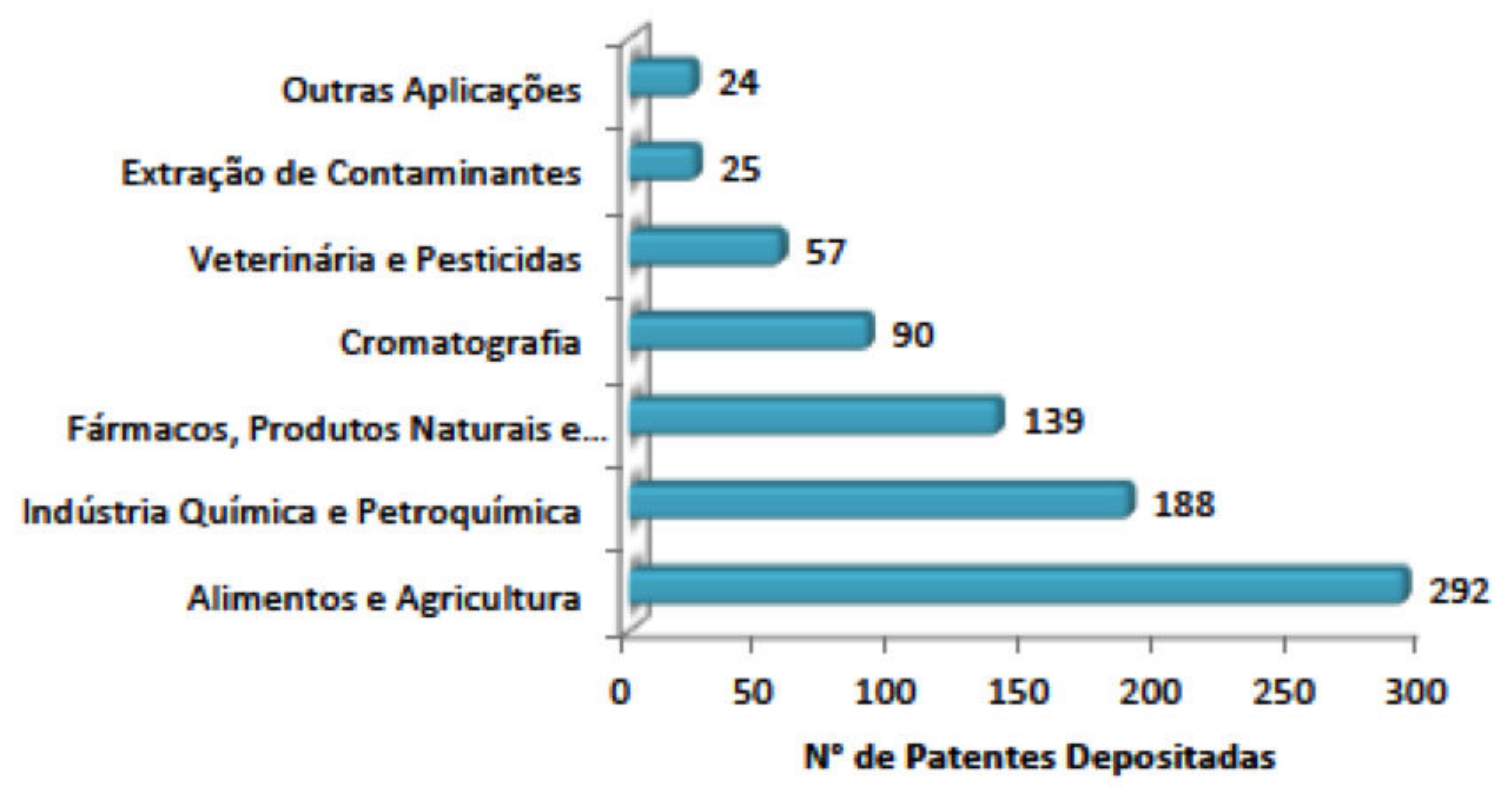

Figura 5. Distribuição das patentes referentes à tecnologia de extração supercrítica por área de aplicação

Grande parte do aumento no número de pesquisas em desenvolvimento e dos depósitos de patentes relaciona-se com as vantagens apresentadas por esta técnica em relação aos métodos convencionais. Entre as tantas vantagens advindas desta tecnologia, destaca-se o fato dos processos envolvidos serem limpos, caracterizando-se assim como uma "tecnologia verde", sem nenhum impacto negativo para o meio ambiente. Observa-se que sua aplicação já faz parte do cenário atual de diferentes países, sendo principalmente impulsionada pela crescente demanda de produtos de alta qualidade e da globalização da economia. Plantas industriais com aplicação prática no comércio de insumos farmacêuticos, alimentícios, químicos e cosméticos já estão disponíveis nos Estados Unidos, Europa e Ásia. Destacase que o aumento da aplicação desta tecnologia na área industrial se deve principalmente pela seletividade, facilidade e capacidade de separação que a técnica permite na obtenção de grande número de compostos orgânicos, os quais muitas vezes são impossíveis ou inviáveis de se extrair pelos processos tradicionais.

\section{Conclusão}

A utilização e correlação dos indicadores de output (documentos de patentes) com os valores em investimentos em PD\&l, considerados como indicadores de input, evidenciaram que os investimentos em PD\&I refletem no número de patentes depositadas. Em relação à tecnologia pesquisada, a extração com fluidos supercríticos, os países desenvolvidos são os principais detentores desta tecnologia, sendo a China o país que lidera o ranking de depósitos. Em relação aos países estudados, o Brasil possui os menores investimentos em PD\&l e consequentemente, o menor número de patentes depositadas tanto na área estudada, quanto em outras áreas tecnológicas.

A utilização da tecnologia de extração supercrítica tem sido bastante estudada e aplicada nas áreas das indústrias farmacêutica, de alimentos, cosmética, de perfumaria e para as indústrias de processamento químico, podendo ainda 
haver mais desenvolvimentos nesses processos devido às crescentes pesquisas na área. Com os resultados desse trabalho é possível notar que novas aplicações vêm surgindo nos últimos anos e os estudos sobre aplicação da tecnologia de extração supercrítica não cessam, assim como o aumento do número de depósito de patentes. Os investimentos em PD\&I são extremamente necessários para a evolução e amadurecimento inovativo e tecnológico de qualquer país. A aplicação correta destes dispêndios financeiros é essencial para aumentar, em longo prazo, o número de depósito de patentes.

\section{Agradecimentos}

Os autores agradecem o Serviço Nacional de Aprendizagem Industrial - Departamento Nacional (SENAI DN) pelo financiamento do projeto (Extração Supercrítica de Compostos Bioativos da Própolis), ao Conselho Nacional de Desenvolvimento Científico e Tecnológico (CNPq) e a Coordenação de Aperfeiçoamento de Pessoal de Nível Superior (CAPES) pelas bolsas de pesquisa.

\section{Referências Bibliográficas}

${ }^{1}$ Santos, R. N. M. Produção Científica: Por que Medir? O que Medir? Revista Digital de Biblioteconomia e Ciência da Informação 2003, 1, 1. [Link]

${ }^{2}$ Contini, E.; Séchet, P. Ainda há um longo caminho para a Ciência e Tecnologia no Brasil. Revista Brasileira de Pós-Graduação 2005, 2, 30. [CrossRef]

${ }^{3}$ Liberal, C. G. Indicadores de ciência e tecnologia: conceitos e elementos históricos. Ciência \& Opinião 2005, 2, 121.

${ }^{4}$ OECD; Eurostat. Oslo Manual: Guidelines for Collecting and Interpreting Innovation Data, 3rd Edition, The Measurement of Scientific and Technological Activities, OECD Publishing, 2005. [CrossRef]
${ }^{5}$ Hayashi, M. C. P. I.; Faria, L. I. L; Hoffmann, W. A. M.; Hayashi, C. R. M.; Ferraz M. C. C. Indicadores de CT\&I no pólo Tecnológico de São Carlos: Primeiras Aproximações. Revista Digital de Biblioteconomia e Ciência da Informação 2006, 3, 17. [Link]

${ }^{6}$ Borges, C. B.; Santos, V. J. B.; Galina, S. V. R. Resultado da inovação em multinacionais estrangeiras - análise de patentes nos BRICs. FACEF Pesquisa 2010, 13, 63. [Link]

${ }^{7}$ Brunner, G. Fractionation of fats with supercritical carbon dioxide. European Journal of Lipid Science and Technology 2000, 102, 240. [CrossRef]

${ }^{8}$ Budich, M.; Brunner, G. Supercritical fluid extraction of ethanol from aqueous solutions. The Journal of Supercritical Fluids 2003, 25, 45. [CrossRef]

${ }^{9}$ Albarelli, J.; Rabelo, R. B.; Santos, D. T.; Beppu, M. M.; Meireles, M. A. A. Effects of supercritical carbon dioxide on waste banana peels for heavy metal removal. The Journal of Supercritical Fluids 2011, 58, 343. [CrossRef]

${ }^{10}$ Shena, B.; Feng, L.; Li, S.; Li, H. Correlating and predicting the solubilities of polycyclic aromatic hydrocarbons in supercritical fluids using the compressed gas model and the reference solubilities. Fluid Phase Equilibria 2013, 337, 100. [CrossRef]

${ }^{11}$ King, M. B.; Boot, T. R.; Extraction of Natural Products Using Near- Critical Solvents, 1a. ed., Blackie Academic \& Professional: Birmingham, 1993. [CrossRef]

${ }^{12}$ Machado, B. A. S.; Pereira, C. G.; Nunes, S. B.; Padilha, F. F.; Umsza-Guez, M. A. Supercritical Fluid Extraction Using CO2: Main Applications and Future Perspectives, Separation Science and Technology 2013, 48, 2741. [CrossRef]

${ }^{13}$ Herrero, M.; Mendiola, J. A.; Cifuentes, A.; Ibáñes, E. Supercritical fluid extraction: Recent advances and applications. Journal of Chromatography A 2010, 1217, 2495. [CrossRef] [PubMed]

${ }^{14}$ Rubio-Rodríguez, N.; Diego, S. M.; Beltra'n, S.; Jaime, I.; Sanz, M. T.; Rovira, J. Supercritical fluid extraction of fish oil from fish by-products: A comparison with other extraction methods. Journal of Food Engineering 2012, 109, 238. [CrossRef] 
${ }^{15}$ Herrero, M.; Cifuentes, A.; Ibáñes, E. Suband supercritical fluid extraction of functional ingredients from different natural sources: Plants, food-by-products, algae and microalgae. Food Chemistry 2006, 98, 136. [CrossRef]

${ }^{16}$ Mattea, F.; Martin, A.; Cocero, M. J. Carotenoid processing with supercritical fluids. Journal of Food Engineering 2009, 93, 255. [CrossRef]

${ }^{17}$ Shojaee, S. A.; Rajaei, H.; Hezave, A. Z.; Lashkarbolooki, M.; Esmaeilzadeh, F. Experimental measurement and correlation for solubility of piroxicam (a Non-Steroidal Anti-Inflammatory Drugs (NSAIDs)) supercritical carbon dioxide. The Journal of Supercritical Fluids 2013, 80, 38, [CrossRef]

${ }^{18}$ Machmudah, S.; Winardi, S.; Sasaki, M.; Goto, M.; Kusumoto, N.; Hayakawa, K. Lycopene extraction from tomato peel byproduct containing tomato seed using supercritical carbon dioxide. Journal of Food Engineering 2012, 108, 290. [CrossRef]

${ }^{19}$ Quintella, C. M.; Meira, M.; Guimarães, A. K.; Tanajura, A. S.; da Silva, H. R. G. Prospecção Tecnológica como uma Ferramenta Aplicada em Ciência e Tecnologia para se Chegar à Inovação. Revista Virtual de Química 2011, 3, 406. [CrossRef]

${ }^{20}$ Machado, B. A. S.; Reis, J. H. O.; Figueiredo, T. V. B.; Druzian, J. I. Mapeamento Tecnológico da Goma Xantana sob o enfoque em pedidos de patentes depositados no mundo entre 1970 a 2009. Gestão, Inovação e Tecnologias 2012, 2, 154. [CrossRef]

${ }^{21}$ Steffani, E.; Tese de Doutorado, Universidade Federal de Santa Catarina, 2003. [Link]

${ }^{22}$ Adams, M. K. Analysis of coffee: production and trade. Allentown: Liquid Carbonic Industries Corporation 1991, 1.

${ }^{23}$ Anklam, E.; Müller, A. Extraction of caffeine and vanillin from drugs by supercritical carbon dioxide. Die Pharmazie 1995, 50, 364. [Link]

${ }^{24}$ Mcnally, M. E. P. Method development in supercritical fluid extraction. Journal of $A O A C$ International 1996, 79, 380. [Link]
${ }^{25}$ OECD. OECD Science, Technology and Industry Outlook 2012, OECD Publishing, 2012. [CrossRef]

${ }^{26}$ Sítio do Ministério da Ciência, Tecnologia e Inovação - Brasil. Disponível em: $<$ http://www.mct.gov.br/index.php/content/ view/740.html?execview=>. Acesso em 10 fevereiro 2014.

${ }^{27}$ Queiroz, O. R.; Dissertação de mestrado, Fucape Business School, 2009. [Link]

${ }^{28}$ Kim, L. Technology Policies and Strategies for Developing Countries: Lessons from the Korean Experience. Technology Analysis \& Strategic Management 1998, 10, 311. [CrossRef]

${ }^{29}$ Niosi, J. Fourth-Generation R\&D: from linear models to flexible innovation. Journal of Business Research 1999, 45, 111. [CrossRef]

${ }^{30}$ Chang, P.; Shih, H. The innovation systems of Taiwan and China: a comparative analysis. Technovation 2004, 24, 529. [CrossRef]

${ }^{31}$ Chiu, K. L.; Cheng, Y. C.; Chen, J. H.; Chang, C. J.; Yang, P. W. Supercritical fluids extraction of Ginkgo ginkgolides and flavonoids. The Journal of Supercritical Fluids 2002, 24, 77. [CrossRef]

${ }^{32}$ Nalawade, S. P.; Picchioni, F.; Janssen, L. P. B. M. Supercritical carbon dioxide as a green solvent for processing polymer melts: Processing aspects and applications. Progress in Polymer Science 2006, 31, 19. [CrossRef]

${ }^{33}$ Wang, L.; Yang, B.; Dua, X.; Yi, C. Optimisation of supercritical fluid extraction of flavonoids from Pueraria lobata. Food Chemistry 2008, 108, 737. [CrossRef]

${ }^{34}$ Temelli, F. Perspectives on supercritical fluid processing of fats and oils. The Journal of Supercritical Fluids 2009, 47, 583. [CrossRef]

${ }^{35}$ Melo, C. I.; Bogel-tukasika, R.; BogelŁukasik, E. Combination of supercritical carbon dioxide and ionic liquid in a novel assembly of carvacrol. The Journal of Supercritical Fluids 2012, 61, 191. [CrossRef] ${ }^{36}$ Gomes, P. B.; Mata, V. G.; Rodrigues, A. E. Production of rose geranium oil using supercritical fluid extraction. The Journal of Supercritical Fluids 2007, 41, 50. [CrossRef] 
${ }^{37}$ Pourmortazavi, S. M.; Hajimirsadeghi, S. S. Supercritical fluid extraction in plant essential and volatile oil analysis. Journal of Chromatography A 2007, 1162, 2. [CrossRef] [PubMed]

${ }^{38}$ Brunner, G. Supercritical fluids: technology and application to food processing. Journal of Food Engineering 2005, 67, 21. [CrossRef]
${ }^{39}$ Lesoin, L.; Crampon, C.; Boutin, O.; Badens, E. Preparation of liposomes using the supercritical anti-solvent (SAS) process and comparison with a conventional method. The Journal of Supercritical Fluids 2011, 57, 162. [CrossRef] 